\title{
EDUCACIÓN PARA LA PAZ Y CULTURA DE PAZ EN DOCUMENTOS INTERNACIONALES
}

\author{
Carmen Labrador \\ Universidad Complutense de Madrid
}

\begin{abstract}
RESUMEN. Se pretende conocer las aportaciones de diferentes organismos internacionales sobre educación para la paz y cultura de paz. Especialmente se han tenido en cuenta documentos del Consejo de Europa y de la UNESCO. La "Declaración de Viena y la consolidación de la paz", la "Declaración de Budapest y la construcción de la ciudadanía", el "Proyecto transdisciplinario por una cultura de paz" y el "Manifiesto 2000" vertebran estas páginas. La "Carta de Auroville" sirve de introducción. A partir de un breve análisis de esta documentación permite entender la educación para la paz como una experiencia de aprendizaje en una sociedad pluricultural que afronta la diferencia de manera informada, razonable, tolerante y ética. El Año Internacional de la Cultura de Paz, constituye una ocasión única para revisiones conceptuales y para decidir que la paz sólo puede lograrse con nuestros comportamientos, nuestras actitudes y nuestro vivir cotidiano.
\end{abstract}

ABSTRACT. The purpose to this paper is to know the contribution the International Organism, specially the Council of Europe and UNESCO, over education to the peace and culture of peace. The Declaration of Vienna, the Declaration of Budapest, the Transdisciplinary Proyect 'Toward: a Culture of Peace' and the Manifesto 2000 for a Culture of Peace and Non-Violence are the documents studied.

The education of peace is considered to learned sperience in the societe pluricultural where the diference understand with tolerance, way of going abaut informed, reasonable and ethics.

At the even of the new millennium, the General Assembly of the United Nations has proclaimed the year 2000 as the International Year for the Culture of Peace and the Decade 2001-2010 as the International Decade for the Children of the World. This is on acount of which invite to conceptual revisions at to decide that the peace only to get with our conduct, our attitude and our experience daily.

\section{Introducción}

El presente artículo forma parte de un trabajo más amplio que pretende estudiar diferentes dimensiones de la educación en documentos emanados de Organismos Internacionales. Para esta ocasión se han tenido en cuenta sobre todo, algunas de las aportaciones más significativas de los últimos años que tienen su origen en el Conse- 
jo de Europa y en la Organización de las Naciones Unidas para la Educación, la Ciencia y la Cultura (UNESCO), si bien las interrelaciones ente ellas son importantes y el apoyo entre estos organismos en materia de educación es fecundo. Las Declaraciones de Viena y de Budapest, el Proyecto Transdisciplinario "Por una cultura de paz", así como el Manifiesto 2000 constituyen las líneas eje de estas páginas.

Naturalmente una formulación del tema de estas dimensiones es difícilmente abordable en los límites que cualquier artículo permite; por esta razón no debe entenderse que pretendemos dar cuenta de modo exhaustivo del importante número de documentos publicados en los últimos años, sino de una pequeña relación de aquéllos que consideramos forman cierta unidad en torno a los objetivos de este artículo. Se da preferencia a determinadas Conferencias Internacionales, Cumbres Mundiales, Declaraciones, etc. que tiene relación con la temática propuesta.

\section{Educación para la paz}

En la 44 reunión de la Conferencia Internacional de Educación celebrada en Ginebra en 1994 y ratificada en noviembre de 1995 por la Conferencia General de la UNESCO, se redactó el documento titulado La educación para la Paz, los Derechos Humanos y la Democracia. En él se dice que la educación para la paz consiste en "fomentar la capacidad de apreciar el valor de la libertad y las aptitudes que permiten responder a sus retos. Esto exige la preparación de los ciudadanos para que sepan manejar situaciones difíciles e inciertas, prepararlos para el ejercicio de responsabilidades individuales. Esto unido al reconocimiento del valor del compromiso cívico, de la asociación con los demás para resolver problemas y trabajar por una comunidad justa, pacífica y democrática"

Unos años más tarde Johan Galtung escribía: "Educar para la paz es enseñar a la gente a encararse de manera más creativa, menos violenta, a las situaciones de conflicto y darle los medios para hacerlo"

La UNESCO cuyo cometido fundacional es "construir los baluartes de la paz en la mente de los hombres" ${ }^{2}$ propicia la cooperación internacional en sus ámbitos de competencia, con la convicción de que los retos del nuevo milenio exigen una reordenación ética, social, económica y política; de que la paz sólo puede forjarse con las herramientas de la paz y que el mundo del futuro ha de alzarse sobre el amor, la tolerancia y la educación. Son palabras del entonces Director General F. Mayor Zaragoza.

Este futuro no podrá construirse y asegurarse con esquemas económicos ni con decisiones políticas, ni con fórmulas prefabricadas, sino mediante principios éticos compartidos y mediante valores que sirvan de estímulo y fundamento a las nuevas generaciones.

1. J. Galtung, La educación para la paz sólo tiene sentido si desemboca en la acción, El Correo de la UNESCO, febrero 1997.

2. En la Carta Constitucional de la UNESCO se lee: "Puesto que las guerras nacen en la mente de los hombres, es en la mente de los hombres donde deben erigirse los baluartes de la paz". 
En todos los documentos se nos recuerda siempre que la construcción de la paz es tarea compleja aunque posible porque nada es inmutable cuando existe un empeño común en transformar la realidad. Con la razón, el diálogo y la comprensión debe lograrse el cambio indispensable, para dejar de invertir en armamento y empezar a invertir en libros, en ordenadores, en medicamentos, en viviendas, en proteger el medio ambiente y en luchar contra la miseria.

Reflexionar sobre lo que podría ser la educación para la paz es una verdadera necesidad y un reto de primera magnitud, especialmente si se tiene la percepción de que la cultura de la violencia está muy presente en nuestra vida cotidiana y afecta a millones de personas de todo el planeta. ${ }^{3}$

Construir una cultura de paz exige no aceptar aquellas conductas sociales que ensalzan el uso de la fuerza y la violencia o que valoran el desprecio y el desinterés por los demás y a la vez insiste en superar determinadas incompatibilidades entre los grupos humanos. En este sentido la educación para la paz aborda el difícil reto de definir criterios adecuados que permitan interpretar correctamente la realidad. Además "supone también garantizar el acceso de la mujer a la educación y posibilitar su autonomía económica, ya que esta igualdad de oportunidades es un requisito previo para lograr los cambios de actitudes y mentalidades de los que depende una cultura de paz". ${ }^{4}$

En julio de 1997 la UNESCO promovió una Cumbre Regional para el Desarrollo Político y los Principios Democráticos, en cuya declaración final "Gobernar la globalización: el consenso de Brasilia" se ponía de manifiesto la necesidad de "un nuevo pacto de gobernabilidad global que debería incluir un nuevo contrato moral por la paz <...> para construir un orden de desarrollo compartido que libere a la humanidad de las ruinas sociales de la pobreza y la disigualdad" ${ }^{\prime \prime}$.

De las reflexiones anteriores se deduce que un buen planteamiento de educación para la paz supone tener en cuanta "la condición de complejidad y diversidad, derivada del propio factor humano, como realidad cotidiana, porque la paz es diversa, plural y polisémica puesto que los pueblos la entienden de formas diferentes."

En cierta medida la educación para la paz es una educación para superar positivamente las tensiones y contradicciones que vive nuestra sociedad, que en el Informe Delors se contemplan como tensión entre lo global y lo local es decir el proceso de convertirse progresivamente en ciudadanos del mundo sin perder las propias raíces; tensión entre universal e individual, entre tradición y modernidad, entre competitividad e interés por la igualdad de oportunidades, entre lo espiritual y lo material; tensión entre la expansión del conocimiento y la capacidad de los seres humanos para asimilarla. ${ }^{6}$

La educación para la paz ha de ser también una educación para el encuentro, el diálogo, la cooperación, la cesión de confianza y un lugar para desarrollar nuestras potencialidades. Si además entendemos la paz como "transformación creativa de conflictos" sus elementos inexcusables son el conocimiento, la imaginación, la compa-

3. V. Fisas, Cultura de paz y gestión de conflictos, Icaria, Barcelona, p 13.

4. V. Fisas, op. cit., p. 360-361.

5. V. Fisas, op. cit., p. 367.

6. J. Delors, La educación encierra un tesoro, UNESCO 1996. 
sión, la solidaridad, la integración y la participación, formando todos ellos el marco adecuado para generar una cultura de paz, opuesta a la cultura de violencia, que permita desarrollar esos valores, y potencialidades y satisfacer las necesidades correspondientes.

No debemos olvidar que mediante la educación pueden introducirse "de forma generalizada los valores, instrumentos y conocimientos que forman las bases del respeto hacia la paz, los derechos humanos y la democracia, porque la educación es un importante medio para eliminar la sospecha, la ignorancia, los estereotipos, las imágenes de enemigo; y al mismo tiempo promover los ideales de paz, tolerancia y no violencia, la apreciación mutua entre los individuos, los grupos y las naciones" ${ }^{\prime \prime}$.

La educación es también el eje dinámico del triángulo formado por la paz, el desarrollo y la democracia, un triángulo interactivo cuyos vértices se refuerzan mutuamente por lo que es "la herramienta que nos permite trascender la condición de individuos y llegar a ser personas, es decir, ciudadanos que aportan a la sociedad, capaces de buscar y expresar la verdad, de contribuir a que las comunidades y las naciones alcancen una vida mejor ${ }^{\prime \prime}$.

Todo esto será mucho más fácil y posible si antes hemos aprendido y practicado el sano ejercicio de "imaginar el futuro". En palabras de Elise Boulding, "es esencial una educación que expanda la capacidad de imaginar un mundo diferente. La imaginación da el poder para actuar en favor del cambio social y para poner en marcha aventuras pacíficas constructivas". ${ }^{9}$

En consecuencia la educación para la paz debe enseñar a perder el miedo a la diferencia de los otros, a tratar a las demás culturas en igualdad de condiciones, no imponiendo los modelos en ningún caso, conscientes de que para ello "es necesario un cambio tal que lleguen a importar más las cosas que puedan ser compartidas por muchos, o mejor por todos, al mismo tiempo que se considera la diferencia entre las cosas tuyas y las mías" ${ }^{\prime 10}$. Naturalmente este proceso requiere un aprendizaje orientado a reconocer los intereses de los otros y a aceptar el hecho de la multiculturalidad íntimamente unido a la riqueza de la diversidad y de la identidad.

En síntesis, la educación para la paz ha de ser un esfuerzo capaz de armonizar las distintas tendencias y de consolidar una nueva manera de ver, entender y vivir el mundo, dando confianza, seguridad y autoridad a las personas y a las sociedades, superando desconfianzas, ayudando a comprender las diferencias, percibiendo la realidad del mundo desde una perspectiva global que pueda ser compartida por todos.

7. Syminides, Janusz; Singh, Kishore, Constructing a culture of peace: challenges and perspectives. An introductory note, en From a culture of violence to a culture of peace, UNESCO, 1996, pp. 20-30.

8. F. Mayor Zaragoza, La nueva página, UNESCO, Círculo de Lectores, 1994, p. 45.

9. E. Boulding, "The Concept of Peace Culture", en Peace and Conflict Issues after the Cold War, UNESCO, 1992, p. 127.

10. A. Aisenson, Resolución de conflictos. Un enfoque psicosociológico, Fondo de Cultura Económica, México 1994, p. 34. 


\section{Los derechos humanos y el derecho a la paz}

En 1997 la UNESCO celebró en Oslo y en Las Palmas reuniones preparatorias de un "proyecto de declaración" del derecho que toda persona tiene a la paz. En esta Declaración aprobada por la Conferencia General se establecía la paz como un derecho y como un deber. En el Foro internacional "Por una cultura de paz y diálogo entre civilizaciones", celebrado en Moscú en mayo de 1999 se afirmaba que "la educación es un derecho fundamental de los ciudadanos, es una de las claves esenciales para construir la cultura de la paz. La educación multilingüe y multicultual para el desarrollo de la tolerancia y el entendimiento entre ciudadanos libres de prejuicios" ${ }^{11}$.

En nuestro caso interesa señalar esta dimensión y considerar "el derecho a la paz" como uno de los pilares fundamentales del discurso sobre la educación para la paz y la cultura de paz. En este sentido palabras más autorizadas que las mías se han expresado con absoluta claridad y competencia. Me refiero una vez más en este artículo a las siguientes afirmaciones:

“...ganar la paz no significa solamente evitar la confrontación armada sino elaborar con tesón y prudencia, los instrumentos que permitan erradicar las causas de la violencia individual y colectiva: la injusticia y la opresión; la ignorancia y la miseria; la intolerancia y la discriminación. Para ganar la paz hay que esforzarse por edificar, sin prisa pero sin pausa, un armazón de valores y actitudes que modifiquen a medio y largo plazo tanto la conducta íntima como la social. Ganar la paz quiere decir consolidar la convivencia democrática en un nuevo empeño de tolerancia y generosidad que es, en última instancia, una tarea de amor". ${ }^{12}$

En la Conferencia Internacional de Educación celebrada en Ginebra en 1994, en la discusión y definición de las directrices comunes de actuación en Educación para la Paz, los Derechos Humanos y la Democracia, se considera de manera relevante la educación para la ciudadanía como realidad indispensable para el logro de la paz, con implicaciones directas vinculadas a cooperación internacional, desarrollo compartido, participación democrática, solidaridad y tolerancia entendidos todos ellos como elementos necesarios para construir una cultura de paz, que se convierta en excelente contexto para la educación misma.

La concepción educativa que subyace en las consideraciones expuestas anteriormente y que corresponden a "un periodo de transición y transformación acelerada caracterizado por la expresión de la intolerancia, las manifestaciones de odio racial y étnico, el recrudecimiento del terrorismo en todas sus formas y manifestaciones, la discriminación, la guerra y la violencia hacia 'el otro' y las disparidades cada vez mayores entre ricos y pobres, tanto en el plano internacional como local y nacional, las estrategias de acción deben apuntar a garantizar las libertades fundamentales, la paz, los derechos humanos y la democracia y a fomentar al mismo tiempo el desarollo económico y social sostenible y equitativo ya que se trata de componentes de la construcción de una cultura de paz. (Esto) exige la transformación de los estilos tra-

11. F. Mayor Zaragoza, International Year for the Culture of Peace 2000, UNESCO, p. 1.

12. F. Mayor Zaragoza, Cultura de Paz, Crítica, Mayo 1994, p. 7. 
dicionales de la acción educativa"13 y sin duda constituye un gran reto para todos los educadores no exentos de responsabilidades al respecto.

El balance de las actividades de los diferentes Organismos Internacionales relacionadas con su compromiso y su labor por la Paz y los Derechos Humanos es positivo y esperanzador. Baste señalar algunas de sus realizaciones:

- En 1993 en Montreal, se presenta el "Plan de Acción Mundial de Educación para los Derechos Humanos y la Democracia"

- En 1993 en Viena, se aprueba la "Declaración y Programa de Acción de la Conferencia Mundial sobre Derechos Humanos"

- La "Conferencia Internacional de Educación de Ginebra" de 1994 insiste sobre el tema.

- El "Plan de Acción para el Decenio de las Naciones Unidas para la Educación en la esfera de los Derechos Humanos" de 1995-2005, es sumamente explícito al respecto.

Existen otras más imposible de reseñar a pesar de su interés.

En todos los documentos pueden apreciarse tendencias significativas que fundamentalmente se concretan en cooperación con los gobiernos respecto a las políticas educativas, en relaciones con las instituciones competentes, en la actuación con programas educativos sobre todo aquéllos que exigen a la vez fuertes dosis de investigación. Temas recurrentes son la promoción de los Derechos Humanos, la lucha contra todo tipo de discriminación, el afianzamiento de los procesos democráticos, el pluralismo cultural y el diálogo intercultural, las acciones preventivas, las actuaciones postbélicas que además son ámbitos indiscutibles, presentes en todos los foros y que reclaman acciones concretas y eficaces, como aparece en los mensajes, reiteradamente expresados, en los documentos en cuestión.

El hecho de que la educación se encuentre en el centro mismo de la cultura democrática, cultura que desarrolla valores de libertad, de autonomía, de progreso social y personal; la convicción de que la tolerancia no excluye sino que integra, no rehusa las particularidades sino que potencia su desarrollo en colectividad, nos da la oportunidad de "aprender a coexistir con nuestro entorno, aprender a coexistir con las demás culturas", que en definitiva son, "los mayores desafíos de este fin de siglo"14.

Por lo que tiene de simbólico, resulta obligada una referencia a Auroville, "la ciudad internacional de la aurora" o "ciudad de las necesidades de la tierra" inaugurada el 28 de febrero de 1968. En la ceremonia de colocación de la primera piedra, representantes de numerosos países depositan tierra de sus respectivos lugares en una urna para representar el acercamiento entre los pueblos del mundo. En el fondo de ésta se

13. Comisión Española de la UNESCO, Proyecto de Plan de Acción Integrado sobre la Educación para la Paz, los Derechos Humanos y la Democracia, Boletín Informativo, no 4, Enero-Marzo 1995, pp. 60-61.

14. F. Mayor Zaragoza, Por una cultura de la democracia, El Correo de la UNESCO, Noviembre 1992, p. 49. 
encuentra la "Carta de la Ciudad" que recoge los principios rectores de esta organización tan peculiar y utópica. En ella leemos:

"Auroville no pertenece a nadie en particular. Auroville, pertenece a la humanidad en su conjunto".

"Auroville será el baluarte de la educación continua, del progreso constante y de una juventud que nunca se marchita"

"Auroville aspira a ser el puente entre el pasado y el porvenir. Aprovechando todos los descubrimientos exteriores e interiores, quiere lanzarse con intrepidez hacia las realizaciones futuras"

"Auroville será el núcleo de la búsqueda material y espiritual que dará un cuerpo viviente a una humanidad unida y concreta"15.

La Conferencia General de la UNESCO de 1970, pide al Director General que tome las medidas necesarias para la promoción del desarrollo de Auroville como importante Programa Cultural Internacional donde se ponen de relieve unos fines determinados que se refieren a la comprensión, la paz y la educación que conduzcan al desarrollo armonioso del individuo y de la sociedad ${ }^{16}$.

Un breve análisis de estos principios nos permite señalar aquellos aspectos que, de modo recurrente aparecen una y otra vez en diferentes escritos. Tienen que ver generalmente con actitudes y comportamientos relacionados con la realización personal y social favorecidas por la cooperación, la comprensión, el conocimiento y el logro de la paz. En definitiva, conseguir que los hombres se relacionen más, se comprendan mejor y se conozcan.

\section{La Declaración de Viena y la consolidación de la paz}

El Consejo de Europa, institución política por excelencia, el 9 de octubre de 1993 aprobaba la Declaración de Viena con el objeto de consolidar la paz y la estabilidad en el continente en los momentos de configuración de la "nueva Europa".

Con ella se pretendía facilitar en los diferentes países el pluralismo democrático, la universalidad de los derechos del hombre y el derecho a un patrimonio cultural enriquecido por la diversidad. Naturalmente, la esperanza de esta Europa no debía ser destruida por ambiciones territoriales, reaparición de nacionalismos agresivos, perpetuación de zonas de influencia, intolerancia y/o ideologías totalitarias. Los esfuerzos de los diferentes gobiernos se orientan en este sentido.

En gran medida los planteamientos del Consejo de Europa adoptan como objetivo contribuir al desarrollo de los desafíos de la sociedad del siglo XXI, desafíos que se resumen en la realización de aquellos valores que definen nuestra identidad europea y en la mejora de la calidad de vida de los ciudadanos. Evidentemente, la creación de una Europa tolerante y próspera exige cooperación entre colectividades locales, regio-

15. La Carta de Auroville, El Correo de la UNESCO, Enero 1993, p. 49.

16. M. A. Fernández García, La UNESCO. Historia y prospectiva, Tesis Doctoral, Universidad de Deusto, 1998, p 360. 
nales e internacionales y se sitúa ante el gran reto educativo del milenio: la educación para la paz.

La cooperación cultural a través de la educación, los medios de comunicación social, la acción cultural, la participación de los jóvenes, la cohesión con relación a la diversidad y la integración de los emigrantes representan esfuerzos comunes en favor de la paz y la democracia. En la perspectiva política las acciones se concretan en mejorar la eficacia de las decisiones de la Convención Europea de Derechos Humanos con relación, sobre todo, a garantizar las libertades fundamentales, proteger a minorías nacionales y luchar contra el racismo, la xenofobia, el antisemitismo y la intolerancia, posibilitar la utilización de instrumentos adecuados para estimular el desarrollo de acciones culturales en las cuales los poderes públicos se asocien con la sociedad civil.

Las implicaciones educativas de estos planteamientos son indiscutibles. Por una parte, favorecen la creación de un clima de tolerancia y diálogo necesarios para la participación de los ciudadanos en la vida política. Por otra, aseguran el respeto a aquellos principios que son la base de nuestra tradición europea común. Es decir:

a) igualdad ante la ley,

b) no discriminación,

c) igualdad de oportunidades,

d) derecho de asociación y de reunión,

e) participación activa en la vida pública.

A los diferentes Estados corresponde crear condiciones de tal naturaleza que permitan a las personas y a los grupos desarrollar su cultura, preservando su religión, sus tradiciones, sus costumbres y su lengua. De ahí la importancia de los acuerdos bilaterales para asegurar la protección de las minorías con sus identidades culturales y estilos de vida.

El Comité de Ministros es el encargado de elaborar medidas de confianza de tal manera que acrecienten la tolerancia y la compresión entre los pueblos. ${ }^{17}$

La Declaración y el plan de acción sobre la lucha contra el racismo, la xenofobia, el antisemitismo y la intolerancia fueron firmados por los reyes y por los primeros ministros de los diferentes países. En el plan de acción, explícitamente, se reafirman los valores de cooperación y solidaridad que hacen posible reducir la marginación y la exclusión social. ${ }^{18}$

Parece oportuno reproducir algunos de los puntos del documento citado por considerarlos de sumo interés para este tema y por su utilidad para elaborar posibles programas de educación para la paz.

Se habla en primer lugar de una "extensa campaña europea para movilizar a jóvenes en favor de una sociedad de tolerancia fundada sobre la dignidad igual de todos

17. Declaración de Viena. Anexo III, p. 5.

18. Declaración de Viena. Anexo III, p. 6. 
sus miembros y contra las manifestaciones del racismo, la xenofobia, el antisemitismo y la intolerancia". ${ }^{19}$

Se propone el desarrollo de la educación en el dominio de los Derechos del Hombre y del respeto a las diversidades culturales mediante programas orientados a eliminar los prejuicios en la enseñaza de la Historia, poniendo en evidencia las influencias mutuas positivas entre diferentes países, religiones e ideas en el desarrollo histórico de Europa._En este sentido debe reseñarse la Conferencia internacional sobre "la enseñanza de la historia para la integración y la paz"20

Aparecen en segundo lugar los aspectos relacionados con la cooperación desde la perspectiva de desarrollo de políticas de lucha contra la exclusión social y la gran pobreza.

Importancia especial se concede a los medios de comunicación social, de tal manera que se solicita a los profesionales de los medios que presenten sus reportajes y comentarios sobre actos de racismo y de intolerancia elaborando códigos deontológicos profesionales que reflejen estas exigencias. ${ }^{21}$

\section{La Declaración de Budapest y la construcción de la ciudadanía}

Con ocasión de la celebración del 50 aniversario del Consejo de Europa el Comité de Ministros reunidos en Budapest en 1999 hacen un Ilamamiento a la responsabilidad del Consejo urgiendo se tomen las medidas necesarias para construir una sociedad más libre, más tolerante y más justa fundada sobre la solidaridad, los valores comunes y un patrimonio educativo enriquecido por la diversidad. A la vez subrayan el papel primordial de la Convención Europea de los Derechos del Hombre y de los instrumentos fundamentales del Consejo de Europa y de las Naciones Unidas para garantizar a los individuos el ejercicio de sus derechos inalienables.

En este contexto, corresponde a la educación reafirmar los valores espirituales y morales que "son la verdadera fuente de la libertad individual, del pluralismo político y de la preeminencia del derecho", principios que forman la base de toda democracia verdadera. En consecuencia la Declaración final y el Plan de acción ponen el acento en la necesidad de desarrollar la educación para la ciudadanía democrática fundada sobre los derechos y responsabilidades de los ciudadanos, insistiendo, una vez más, en la "imperiosa necesidad de reforzar la conciencia y la comprensión hacia los individuos, hacia sus derechos y sus responsabilidades con el fin de que sean capaces de ejercer estos derechos y de respetar los derechos de los demás".

19. 1995 fue declarado Año Internacional de la Tolerancia. La UNESCO preparó trabajos muy interesantes, entre otros una exposición de dibujos de niños de todo el mundo con representaciones verdaderamente expresivas de la significación que para cada uno de ellos tenía la idea de "tolerancia".

20. El documento principal de la Conferencia: La Historia, instrumento para la paz, es de enorme interés. La Conferencia se celebró en Cartagena de Indias (Colombia) los día 24 al 26 de noviembre de 1996, promovida por el Convenio Andrés Bello-UNESCO.

21. Consejo de Europa, Declaración de Viena, Anexo III, p. 7. 
Naturalmente, también subrayan el papel fundamental de la educación en la promoción de la participación activa de todos en la vida democrática en los diferentes niveles local, regional y nacional.

En este interesante documento se reconocen las orientaciones del Consejo de Europa en todos aquellos sectores implicados en la promoción de una ciudadanía responsable en la sociedad democrática. Como consecuencia, la preocupación por el desarrollo de la violencia, de la xenofobia, del racismo, de los nacionalismos agresivos y de la intolerancia religiosa, que constituyen una grave amenaza para el afianzamiento de la paz y de la democracia, tanto en el plano nacional como en el internacional, es evidente. ${ }^{22}$

Conscientes de la responsabilidad de las generaciones presentes y futuras insisten en que "la educación para la ciudadanía democrática" es condición indispensable para la paz, fundamentada en los derechos y deberes de los ciudadanos.

La educación así entendida se convierte en una experiencia de aprendizaje a lo largo de la vida y en un proceso participativo desarrollado en contextos varios, es decir, en el seno de la familia, en las instituciones educativas, en los lugares de trabajo, en el marco de las organizaciones profesionales, gubernamentales y no gubernamentales, en las colectividades locales, a través de actividades recreativas y culturales y por los medios de comunicación, así como con las diferentes actividades de protección y de mejora del entorno natural y social.

Intenta fomentar una cultura de los derechos del hombre susceptible de asegurar el pleno respeto a estos derechos y la toma de conciencia de las responsabilidades que de ellos se derivan.

Prepara a la población para vivir en una sociedad pluricultural y a afrontar la diferencia de manera informada, razonable, tolerante y ética. Refuerza la cohesión social, la comprensión mutua y la solidaridad y debe incluir todos los grupos de edad y todos los sectores sociales. En definitiva, capacita a los hombres y a las mujeres para desempañar un papel activo en la vida pública y para realizar responsablemente sus propios destinos y los de la sociedad..$^{23}$

A lo largo del documento se subraya la importancia de cuantas actividades se realicen a favor de la educación para la ciudadanía democrática orientadas por los planes de acción de las diferentes Cumbres. ${ }^{24}$

En el mismo informe se estima la posibilidad de acciones suplementarias si son necesarias para poner en marcha la educación para la ciudadanía democrática bien fundamentada, con sentido de permanencia y en todos los niveles y todos los dominios. En este sentido se invita a los Estados miembros:

- a promover la ciudadanía democrática sobre la base de la salvaguarda y del desarrollo de los derechos del hombre y de las libertades fundamentales,

22. En 2001 se celebrará en Sudáfrica la Conferencia Mundial contra el racismo, la discriminación racial, la xenofobia y las formas conexas de intolerancia.

23. Consejo de Europa, Declaración de Budapest, p. 10-11.

24. Especialmente en el marco del plan de acción de la Cumbre de Strasburgo. 
- a hacer de la educación para la ciudadanía, fundada sobre los derechos y las responsabilidades de los ciudadanos, una componente esencial de todas las políticas y prácticas en materia de educación, de formación, de cultura y de juventud.

Del apartado relativo a las decisiones conviene señalar algunos puntos. En primer lugar, la prioridad que se concede a la educación para la ciudadanía en el programa de trabajo del Consejo de Europa, como paso previo y simultáneo para la construcción y el mantenimiento de la paz.

En segundo lugar adoptar el Programa y ponerlo en práctica de manera coordinada con los sectores pertinentes del Consejo de Europa. ${ }^{25}$

El Programa insiste en la aplicación de las diferentes propuestas en ámbitos de educación no formal, informal, formal y en formas de educación continua y educación para todos. Son, sin duda, aportaciones dignas de tener en cuenta en las reflexiones pedagógicas de carácter prospectivo.

Por último, se expresa el deseo de que para todos los programas se adopten hasta el año 2000, las propuestas hechas por las Conferencias de Ministros especializados en estos ámbitos y se tengan en cuenta los principios, las directrices y las recomendaciones de los Estados miembros.

\section{Hacia una cultura de paz}

La idea de construir una "cultura de paz" ha cumplido diez años. Desde que en 1989 la UNESCO emprendiera numerosas actividades en torno a ella se han logrado objetivos importantes y sobre todo se ha conseguido un lugar en la reflexión del sistema de las Naciones Unidas, Organismo que proclamó el año 2000 como Año Internacional de la Cultura de Paz.

Educación para la paz y cultura de paz son formas que se implican mutuamente de tal manera que la cultura de paz es el humus, el contexto sin el cual no es posible la educación y a su vez la educación para la paz es imprescindible para crear esa cultura tan deseada. En algunos de los documentos estudiados para este artículo una y otra expresión se utilizan indistintamente.

Fue en el Congreso de Yamusukru sobre la Paz en la Mente de los Hombres (Côte d'Ivoir 1989) cuando se sentaron las bases de una cultura de paz. Unos años más tarde en febrero de 1994, el Foro Internacional sobre la Cultura de Paz que se celebró en San Salvador fue el punto de partida. Posteriormente, en noviembre de 1997, la Asamblea General de las Naciones Unidas proclamó el Año 2000, Año Internacional de la Cultura de Paz. El Secretario general, M. Kofi Annan, hizo sonar el carillón del "Reloj de la Paz" en el curso de la ceremonia de inauguración oficial que tuvo lugar en París el martes 14 de septiembre de 1999 a las 10 horas.

El Reloj de la Paz es un regalo de la Asociación de las Naciones Unidas de Japón realizado con monedas fundidas ofrecidas por pueblos de más de sesenta países. Tradicionalmente se hace sonar por el Secretario general de la ONU con ocasión del Día

25. Programa adjunto a la Declaración. 
internacional de la paz, que se celebra todos los años en la fecha de apertura de la Asamblea general de las Naciones Unidas. La proclamación del Año internacional se hizo con diversos actos que tuvieron lugar en París y en otras ciudades de todo el mundo. En ésta como en otras ocasiones la UNESCO coordina y auspicia las actividades correspondientes en el todos los países.

La cultura es un componente esencial en la vida y en general en el desarrollo que encarna el bagaje del pasado y se conjuga con el presente. Es además un ingrediente de la comunicación y de la convivencia intra e internacional y sus diferencias enriquecen la vida y potencian el desarrollo equilibrado entre identidades e innovaciones y se generan situaciones de pluralismo que sólo pervive y se engrandece en democracia y paz.

A este respecto la finalidad de la UNESCO, como promotora del progreso cultural consiste en "poner de relieve a través del pluralismo cultural", aquello que acerca a todos los hombres, es decir, aquello que en cada cultura alcanza un nivel universal. Para lograrlo hay que entender el principio o la idea fundamental de que cada cultura -que a su vez es con frecuencia crisol de otras culturas- posee un carácter propio, una "marca registrada" en la historia de la civilización humana, un signo de identidad que le permita reconocerse a sí misma a lo largo de su trayectoria y gracias al cual el resto del mundo también la reconoce <...>

$<\ldots>$ "La misión prioritaria de la UNESCO en el plano cultural consiste naturalmente -según los términos mismos de su Constitución- en "desarrollar e intensificar las relaciones entre sus pueblos, a fin de que éstos se comprendan mejor entre sí y adquieran un conocimiento más preciso y verdadero de sus respectiva vidas", es decir aprendan a convivir en paz.

Su acción cultural apunta, en el fondo, a promover los encuentros y reuniones de artistas, artesanos, intelectuales, pintores, educadores, arquitectos, escritores y poetas de todos los países y regiones, así como a difundir los debates y conclusiones de esas reuniones. Estos hombres y mujeres, representan la principal riqueza de la humanidad; pueden esclarecernos el sentido del pasado, pero también y sobre todo las configuraciones posibles del porvenir. En todos los casos la UNESCO elabora y realiza sus programas culturales con la colaboración de Organizaciones No Gubernamentales y de asociaciones de profesionales, artistas y creadores". ${ }^{26}$

Merece recordarse en este tema el Decenio Mundial para el Desarrollo Cultural, y la representación que el artista Hans Enri realizó para la ocasión. En el emblema aparecen "Cinco rostros de cinco continentes diferentes que encarnan la tierra y simbolizan la multiplicidad creadora de la vida en común, social y culturalmente". ${ }^{27}$

La idea de cultura que recogen los objetivos del Decenio es importante; lo que pretende es conseguir "una diversidad que una", "una creatividad que aproxime" y "una solidaridad que libere", expresiones todas ellas que hablan por sí mismas y no necesitan comentario alguno.

26. Entrevista a F. MAYOR ZARAGOZA, El Correo de la UNESCO, Noviembre 1989, p. 7.

27. Son una expresión de la alegría de vivir y se alzan a la luz del sol cuyo resplandor representa la obra múltiple que lleva a cabo la UNESCO. 
Desde otra perspectiva, se busca integrar la dimensión cultural en el desarrollo (relación entre economía y cultura), afirmar y enriquecer las identidades culturales (estímulo y potenciación de las capacidades, talentos e iniciativas), ampliar la participación en la vida cultural, fomentar la cooperación cultural internacional y devolver a los valores culturales y humanos el lugar central que les corresponde en el desarrollo tecnológico y económico.

A los representantes de las diferentes religiones se les recomienda insistentemente que fundamenten la enseñanza en el amor, el compañerismo y la tolerancia y que renuncien a la mentalidad de "guerra santa" todavía presente en los debates sociorreligiosos actuales.

No debe eludirse el tema de los conflictos que, sin duda, están presentes y pueden ser fuente de creatividad y cambio, razón que justifica el objetivo del programa de cultura de Paz de la UNESCO cuya propuesta no es eliminarlos sino canalizarlos de manera no violenta. El proyecto de cultura de paz es en definitiva un instrumento útil para movilizar a los pueblos en función de su propia transformación y la de su entorno.

\section{El Programa "Cultura de Paz de la UNESCO"}

Cincuenta años después de la fundación de las Naciones Unidas y de la UNESCO, el mundo se encuentra nuevamente en posición de transformar la cultura predominante de violencia en una cultura de paz, entendiendo la "paz como quehacer, como tarea de todos y no simplemente como ausencia de guerra"

El objetivo de una cultura de paz es asegurarse de que los conflictos inherentes a las relaciones humanas se resuelvan de manera no violenta, basándose en los valores tradicionales de la paz y programando acciones globales para su instauración.

La cultura de paz está basada en los principios establecidos en la Carta de las Naciones Unidas, en el respeto por los derechos humanos, la democracia, la tolerancia, la promoción del desarrollo y la educación para la paz.

Con diferentes celebraciones se recordaba al mundo la necesidad de sumar esfuerzos para conseguir ese bien tan escaso. Los llamamientos se sucedían y también las respuestas; baste citar una de ellas:

"Recibimos con gran complacencia la resolución aprobada por la Asamblea General de las Naciones Unidas el pasado 12 de julio, mediante la cual decidió proclamar la Semana Mundial de la Paz, que comenzará el 24 de octubre próximo (1995) en solemne celebración del cincuentenario de las Naciones Unidas. En armonía con tan trascendental resolución, se afirmaron la total disposición de nuestros Gobiernos de dar la más amplia publicidad y asistencia al lanzamiento de dicha proclamación, que, de manera indudable, coadyuvará al establecimiento de un periodo de paz universal. Destacamos la importancia de impulsar una cultura de paz en lberoamérica, que promueva los valores del diálogo y el entendimiento". ${ }^{28}$

28. Declaración de la V Cumbre Iberoamericana de San Carlos de Bariloche. Argentina 16-17 de octubre de 1995. Tercera parte, p. 11. 
Ya en 1993 los ministros de educación de Iberoamérica pusieron en "el primer plano de las decisiones políticas de la nueva década, la urgencia de lograr una educación pertinente para un desarrollo que compatibilice crecimiento económico, equidad social y democratización política... "porque no hay desarrollo sustentable sin paz, no hay paz sin desarrollo, no hay paz ni desarrollo sin democracia y nada de esto podremos alcanzar plenamente sin asegurar el derecho de la niñez a una educación de calidad para todos".

Septiembre de 1999 es un mes clave para el proyecto de cultura de paz. La Asamblea General de Naciones Unidas adopta por consenso una resolución relativa a la Declaración y al Programa de acción con el fin de que los gobiernos, las organizaciones internacionales y la sociedad civil puedan inspirarse constantemente en sus disposiciones, en su acción y promover una cultura de paz en el milenio que va a comenzar. ${ }^{29}$

Según este texto la cultura de paz puede definirse "como el conjunto de valores, actitudes, tradiciones, comportamientos y modelos de vida, fundados sobre el respeto a la vida, el rechazo a la violencia y la promoción y la práctica de la no violencia por la educación, el diálogo y la cooperación..."

El acceso a la educación y a varias formas de aprendizaje es un fin necesario pero no condición suficiente para una cultura de paz que exige además:

- Implementar planes nacionales de educación y programas que contengan la educación para una cultura de paz.

- Alentar mejoras en los objetivos e innovación del currículo, contenidos y métodos de enseñanza, materiales pedagógicos y también preparación de profesores y otras personas dedicadas a la educación.

- Impulsar la cooperación bilateral y multilateral y revisar los textos de historia y geografía.

- Promover la diversidad lingüística en todos los niveles de la educación.

- Potenciar la educación física y deportiva por una cultura de paz.

- Defender la paz, los derechos humanos y la democracia.

Los diferentes Organismos Internacionales han mostrado con energía su interés por llevar a buen término etas propuestas.

\section{Educar para una cultura de paz}

Asistimos al nacimiento de una sociedad mundial, sociedad cuya nota más carácterística es la globalización. Entre sus exigencias se descubre la necesidad de superar determinadas tensiones inherentes al mismo proceso, es el caso de la tensión entre lo global y lo local, superación que precisa nuestra conversión paulatina en ciudadanos del mundo sin perder nuestras raices y participando activamente en la vida de la nación y de la comunidad.

29. Asamblea General de Naciones Unidas. Sesión de clausura de la sesión 53, septiembre 1999, p. 2. 
En este proceso a la educación le corresponde una tarea importante que se convierte a su vez en un gran desafío, porque prepararse para vivir en este mundo globalizado significa "ayudar a transformar una interdependencia de hecho en una solidaridad deseada" que permita a la humanidad "progresar hacia los ideales de paz, de libertad y de justicia social al servicio de un desarrollo humano más armonioso, más auténtico, con el finde hacer retroceder la pobreza, la exclusión, las incomprensiones, las opresiones, las guerras".

La educación debe desarrollar los valores de la solidaridad, basada en el conocimiento y en el respeto de la diversidad que enriquece la globalidad y que es opuesta a la competitividad.

Considerar la globalidad sólo desde la perspectiva económica es reducirla a aqueIlos aspectos menos trascendentes y hasta peligrosos. No debemos olvidar que lo más importante es la dimensión humana, la comprensión entre los pueblos orientada a la construcción de un destino común e interdependiente. De esta manera, la dinámica que se establece entre ellos constituye el marco en el que se desenvuelve la vida de los ciudadanos con exigencias ineludibles de educación de calidad. Porque la "violencia" no está sólo en las confrontaciones bélicas sino que una de las formas de violencia que más se experimentan actualmente es la de no dar oportunidad de calidad a los que tienen menos, puesto que esa violencia los condena siempre a la marginalidad y a la pobreza.

Podría afirmarse que el objetivo número uno de la UNESCO, construir una cultura de paz, requiere la participación de todos, de manera que la prioridad de la Organización para los años 2000-2001 es garantizar a cada persona los medios que le permitan asumir su destino a través de un mayor y mejor acceso a una educación más pertinente, compartir el conocimiento científico y la investigación, la defensa de la libertad de prensa y la protección de nuestro patrimonio cultural.

Construir la paz significa prevenir los conflictos en su origen, actuar mucho antes de que se produzcan esas situaciones tan lamentables y tan frecuentes. Los diferentes sectores de la UNESCO se movilizarán a comienzos del milenio para estimular y valorar todas las iniciativas individuales y colectivas que desarrollan el espíritu de la paz dentro de las sociedades. La cultura de paz solamente podrá existir en la medida en que se le permita a la población tomar esta responsabilidad.

La idea de educar para una cultura de paz ha progresado en el siguiente sentido. No se trata de una cultura definida por la ausencia de conflictos, ni se considera que sea asunto de competencia principal de los Estados sino que es ante todo "una cuestión de valores, de actitudes, de comportamientos individuales y colectivos que fundamentan y encarnan 'el espíritu de paz', según Françoise Riviere, que dirige la Unidad de una Cultura de Paz de la UNESCO.

La educación para una cultura de paz tiene su fundamento en el mandato constitucional de la UNESCO: "Puesto que las guerras nacen en la mente de los hombres, es en la mente de los hombres donde deben erigirse los baluartes de la paz"

En los últimos años la UNESCO y las Naciones Unidas han desarrollado un número de instrumentos, declaraciones y planes de actuación aceptados por la comunidad internacional que proveen del marco para promocionar el concepto de educación 
para una cultura de paz en todo el mundo. Desde 1989 hasta 1999 se han producido 43 Declaraciones sobre la Cultura de Paz, la primera en Côte d'Ivoire, firmada el 1 de julio y la última en París el día 7 del mismo mes; los escenarios de todas las demás se localizan en todo el mundo. ${ }^{30}$

Además y sin ánimo de exhaustividad:

- Recomendación concerniente a la Educación para el Entendimiento Internacional, Cooperación y Paz y Educación relativa a los Derechos Humanos y Libertades fundamentales (París 1974),

- Plan Mundial de Acción en Educación para los Derechos Humanos y Democracia (Montreal 1993),

- Declaración y Programa de Acción de la Conferencia Mundial en Derechos Humanos (Viena 1993),

- Declaración y Marco Integrado de Acción en Educación para la Paz, los Derechos Humanos y la Democracia (París 1995),

- Cumbre Mundial sobre Desarrollo Social de Copenhague 1995

- Plan de Acción de las Naciones Unidas para la Década de los Derechos Humanos y la Educación (1995-2005).

Ante el nuevo milenio, la Asamblea General de las Naciones Unidas ha proclamado el año 2000 como Año Internacional de la Cultura de Paz y la Década 20012010 como Década Internacional para una Cultura de Paz y no Violencia para los niños del mundo. Con ello se pretende conseguir un marco que permita al pueblo movilizarse para un cambio fundamental, porque el acceso a la educación y a varias formas de aprendizaje es un fin necesario pero no una condición suficiente para una cultura de paz. El planteamiento educativo puede hacerse teniendo en cuenta las notas características del Manifiesto 2000 que indicamos en otro apartado.

\section{Proyecto transdisciplinario "Hacia una cultura de paz"}

Para responder al desafío de la construcción de la paz contenido en la Agenda para la Paz publicada por la ONU en 1992 y a la Ilamada urgente lanzada por los Estados miembros en la sesión 140 del Consejo Ejecutivo en la que piden reiterar su compromiso hacia su mandato inicial, la UNESCO ha puesto en marcha en 1994 el Programa por una cultura de la paz.

Este programa es una iniciativa dinámica de construcción de la paz destinada a contrarrestar y a transformar la violencia que predomina ahora en nuestras sociedades.

El Plan a medio plazo de la UNESCO para 1996-2001 insiste en la necesidad imperiosa de establecer una cultura de la paz. A este efecto el plan prevé la puesta en marcha de un poryecto transdisciplinar titulado "Hacia una cultura de paz"

En principio conviene precisar el significado de "cultura de paz" porque las manifestaciones contrarias a la paz revisten formas distintas y variadas, desde la falta de respeto por los derechos humanos, por la justicia y por la convivencia democrática hasta

30. Declaraciones sobre la Cultura de Paz, UNESCO 2000, p. 1-3. 
la pobreza y la ignorancia. Todo esto supone un esfuerzo generalizado para modificar mentalidades y actitudes con deseo explícito de promover la paz y a la vez exige:

- Prevenir los conflictos que puedan engendrar violencia.

- Transformar las condiciones negativas para el entendimiento y la convivencia.

- Restaurar la paz y la confianza en poblaciones que emergen de la guerra o de situaciones conflictivas.

En consecuencia, el concepto de cultura de paz se ha configurando progresivamente al amparo de un vasto movimiento sociopolítico que cuenta con interlocutores del sistema de las Naciones Unidas y de otras instancias numerosas y diversas.

Construir una cultura de paz pretende ser una respuesta a ese conjunto de amenazas y conflictos, una búsqueda de soluciones que no pueden imponerse desde el exterior sino que han de provenir de la propia sociedad. Esta respuesta se ha convertido en un movimiento mundial que busca soluciones, siempre conscientes de que éstas dependen de la intervención conjunta y en frentes muy diversos de hombres y mujeres de todos los sectores de la sociedad.

En todos esos ámbitos la cooperación entre países puede aportar la estabilidad y la ayuda necesarias para obtener resultados duraderos. Fundar una cultura de paz es una empresa que rebasa la responsabilidad de un sector, una comunidad, una región o una nación concretos para adquirir carácter universal. Porque, naturalmente, la cultura de la paz no es sólo una idea, es un movimiento mundial que tiene lugar ahora y que desarrolla numerosas actividades que se articulan en la organización como un gran abanico. Existen proyectos en Angola, Burundi, El Salvador, Etiopía, Haití, Liberia, Malí, Mozambique, Filipinas, la Federación de Rusia, Somalia, la antigua Yugoslavia...

En estos proyectos se pretende:

- Realizar actividades con parlamentarios y demás representantes electos vinculados a consideraciones fundamentales del ejercicio de poder, la democracia y la justicia social.

- Buscar la emancipación de las mujeres para permitirles participar eficazmente en los procesos de la vida social.

- Implantar y fortalecer medios de comunicación que contribuyan a promover una cultura de paz.

- Aplicar programas de educación cívica, de formación para la gestión de conflictos y el ejercicio de la autoridad.

- Promover los ideales democráticos de participación social, de cooperación y de solidaridad.

La UNESCO es consciente de la necesidad de buscar fuera de sus propias estructuras otros interlocutores capaces de prestar apoyo a las iniciativas a favor de la cultura de paz, ya sean gubernamentales o no gubernamentales e intergubernamentales que ya trabajen en este campo.

Siempre y desde esta perspectiva, la educación es considerada como única solución. La educación es la punta de lanza -se dice con frecuencia- de las actividades de promoción de una cultura de paz. La Organización lleva años con proyectos que inviten a sus miembros a incorporar en los programas de educación, formal, informal 
y no formal políticas y principios de acción favorables a la ciudadanía democrática y los derechos humanos. Se han concebido programas especiales que insisten en la educación para la paz y promueven la enseñanza de la tolerancia y la no violencia. ${ }^{31}$

El Proyecto Transdisciplinario "Hacia una Cultura de Paz", busca promover los valores, actitudes y conductas en todos los planos de la sociedad para que se encuentre una solución pacífica a los numerosos problemas que se viven en la sociedad de nuestro tiempo.

El hecho de ser un proyecto transdisciplinar exige que cada sector de la Organización participe activamente en el desarrollo de programas innovadores y de actividades que se encargan de enraizar esta nueva cultura. Es importante tener en cuenta que en este proyecto colabora un número significativo de personas e instituciones que entre sí integran un nuevo movimiento global que pretende una cultura de paz basada en los "principios enunciados en la Carta de las Naciones Unidas y en el respeto a los derechos humanos, la democracia y la tolerancia, la promoción del desarrollo, la educación para la paz, la libre circulación de información y la mayor participación de la mujer como enfoque integral para prevenir la violencia y los conflictos, y que se realicen actividades encaminadas a crear condiciones propicias para el establecimiento de la paz y su consolidación" La UNES$\mathrm{CO}$ es el organismo que encabeza el nuevo movimieto global en pro de una Cultura de Paz.

\section{El diálogo entre culturas}

Dado el carácter pluralista de nuestra sociedad, el tema presenta un interés especial, interés por sí mismo y porque el 2001 será el "Año Internacional del diálogo entre civilizaciones", con el propósito de promover el diálogo como actitud con las implicaciones pedagógicas que tiene.

La Conferencia General en 1987 aprobó el proyecto "Estudio integral de las Rutas de la Seda: Rutas del Diálgo" ${ }^{\prime 32}$. Y fue en 1990 cuando la primera expedición del Estudio Integral de las Rutas de la Seda, recorrió las extensiones desérticas del Oeste de China con objeto de estudiar durante seis años los intercambios que se producían en el transcurso de estas rutas y sus repercusiones sobre las civilizaciones de Oriente y Occidente. A esta primera experiencia siguieron las Rutas del Esclavo, del Hierro, del Pacifico, los espacios del Barroco, etc. En todas ellas se estaban consiguiendo aprendizajes multiculturales con planteamientos innovadores y sobre todo con resultados eficaces. Era exigencia y consecuencia a la vez del pluralismo cultural y promovía el entendimiento mutuo y el respeto entre las diferentes culturas. En la práctica se tra-

31. El Plan de Escuelas Asociadas (PEA) de la UNESCO, creado en 1953, es una red deestablecimientos escolares de todo el mundo que impulsa la educación de niños y jóvenes para el entendimiento internacional y la tolerancia. Interviene en el proyecto de cultura de paz con un doble objetivo: promover una enseñanza de calidad y contribuir al desarrollo de una cultura de paz y no violencia.

LINGUAPAX es otro proyecto también de la UNESCO, promueve la enseñanza de lenguas maternas y lenguas nacionales y extranjeras para la búsqueda d ela paz y la defensa de los derechos humanos.

32. Rutas de la Seda: Rutas del Diálogo 1987-1997, tesis doctoral citada, p. 384. 
ducía en el ejercicio de la comunicación, del diálogo intercultural "todos los días de la vida", así formulado exactamente. ${ }^{33}$

En el desarrollo de la cultura en la comunidad, en el barrio, en el medio rural y también de la cultura urbana, es decir se buscaba la integración entre sociedad y cultura desde la música, el urbanismo, las diferentes manifestaciones populares, la atención a los múltiples cambios. ${ }^{34}$

Son numerosas las actividades culturales de la UNESCO, además de las citadas anteriormente, que incorporan el proyecto de cultura de paz, y fomentan el pluralismo cultural y el diálogo entre las diferentes culturas. Entre ellas pueden señalarse el proyecto "Diálogo intercultural Este-Oeste en Asia Central", el proyecto "Las Rutas de la Fe" y el proyecto "Las Rutas de Al-Andalus"

En definitiva, "el proyecto de cultura de paz aspira a armar a las poblaciones no con fusiles sino con capacidad de diálogo y de entendimiento". Para conseguir este objetivo es fundamental establecer relaciones basadas en la tolerancia y la solidaridad entre poblaciones separadas por diferencias culturales.

En el contexto del proyecto, los usos tradicionales que pueden contribuir a la paz se siguen estudiando, apoyando y considerando como elementos esenciales para la consolidación de la paz y el desarrollo. No en vano el proyecto de cultura de paz propugna la justicia y la igualdad de oportunidades para todos, especialmente para las minorías, las poblaciones indígenas, los refugiados y los desplazados.

También se alienta a los artistas y a otras personas que participan en actividades culturales a ejercer la mayor influencia posible sobre las poblaciones, poniendo su talento al servicio de la paz.

Para transmitir el mensaje de paz los medios de Comunicación Social desempeñan un papel fundamental. No se discute la poderosa influencia que ejercen sobre las mentalidades y las normas de conducta en las diferentes sociedades y su contribución generalmente decisiva al ejercicio de la libertad de opinión y de información. Teniendo en cuenta que el acceso a una información clara y exacta es imprescindible para la promoción de una cultura de paz, ayudar a los medios de comunicación se convierte en tarea ineludible.

Crear un espacio de intercambio de conocimientos y experiencias, fomentar la no violencia y poner las comunicaciones al servicio de la consolidación de la paz y hacerlo en libertad es el mejor camino para la convivencia democrática y en paz y ayuda excelente para promover los derechos humanos y la democracia.

La UNESCO sigue fomentando la elaboración de estrategias nacionales de enseñanza formal y no formal que sirvan para consolidar la paz y sensibilizar a la opinión pública sobre la necesidad de adoptar nuevas mentalidades y normas de conducta en aras de la paz. La información es rica, sugerente, aplicable y generalizable a distintos contextos y al tratamiento de conflictos muy diferentes. La dificultad está sobre todo

33. Roads of dialogue and intercultural echange, UNESCO, Programmes and Priorityes 1994-1995, p. 43.

34. La Década de los pueblos indígenas del mundo, fue otra iniciativa multicultural. 
en como se adquieren los compromisos oportunos y eficaces. Estamos de nuevo ante una demanda formal de educación.

En estrecha colaboración con el Comité Consultivo sobre Educación para la Paz, los Derechos Humanos, la Democracia, el Entendimiento Internacional y la Tolerancia, la Organización fomenta la incorporación de innovaciones a los programas y contenidos educativos así como la mejora de los métodos de enseñanza. Concretamente la División de los Derechos Humanos, la Democracia y la Paz secunda los esfuerzos de los Estados Miembros en estos ámbitos, ayudándoles a elaborar y aplicar programas nacionales de enseñanza sobre derechos humanos, sobre participación democrática, sobre tolerancia y solidaridad.

Se presta especial atención a la evaluación de los planes nacionales para la enseñanza en estos campos. A tal efecto se lleva a cabo una investigación con la Oficina Internacional de Educación sobre leyes, políticas y estrategias nacionales en materia de educación para los derechos humanos en el ámbito universitario en aras de la paz y de la tolerancia y se promueven acuerdos nacionales que permitan desarrollar las enseñanza en este ámbito. Acuerdos que además pueden interesar no sólo a educadores sino a periodistas, productores de programas informáticos didácticos, parlamentarios y otros responsables de la planificación política.

Una concepción abierta y activa de la diversidad es condición imprescindibles para que exista una cultura de paz que además sólo puede tener éxito en un contexto de entendimiento mutuo y de aceptación individual y social.

Consciente de esta realidad la UNESCO tiene la intención de reforzar las redes regionales de promoción de la tolerancia y crear otras redes nuevas. Para ello prepara material didáctico y diferentes instrumentos pedagógicos centrados en la tolerancia y contribuye a su difusión a gran escala, forja nuevas relaciones de colaboración en el campo de la educación para la tolerancia, intenta que los programas sobre la tolerancia hallen eco entre un número creciente de jóvenes y elabora en colaboración con medios de comunicación locales, programas audiovisuales sobre la tolerancia...

Para prestar especial atención a las mujeres se han incorporado las diferencias de género al proyecto transdisciplinario "Cultura de Paz" de tal manera que, entre las prioridades del subproyecto "Las mujeres y la cultura de paz" pueden señalarse al menos tres acciones significativas como son:

- Apoyar las iniciativas de mujeres a favor de la paz.

- Emancipar a las mujeres para que puedan participar democráticamente en el proceso político y ganar así presencia e influencia, especialmente en materia de economía y seguridad.

- Promover en los acuerdos de colaboración, una socialización que tenga en cuenta las especificidades femeninas y una formación para la no violencia y la igualdad, dirigida sobre todo a los niños y a los jóvenes. ${ }^{35}$

35. UNESCO, Proyecto Transdisciplinario "Hacia una Cultura de Paz", p. 4. 


\section{Manifiesto 2000, para una cultura de paz y no violencia}

El "Manifiesto 2000" para una cultura de paz y de no violencia, creado por un grupo de Premios Nobel, reunidos en París con motivo del quincuagésimo aniversario de la Declaración Universal de los Derechos Humanos, traduce las resoluciones de las Naciones Unidas que año tras año se producen.

El Manifiesto 2000 se hizo público el el 4 de marzo de 1999 en París en una conferencia de prensa internacional que se celebró en la Torre Eiffel en la que se invitó a firmar a todos los ciudadanos del mundo. Asistieron cien jóvenes que se comprometieron a difundir el mensaje por todos los países. No es un llamamiento, ni una petición dirigida a instancias superiores, se trata de un compromiso individual y personal.

“En el siglo que ahora está acabando, decía el Director General de la UNESCO, se han producido extraordinarios progresos en la ciencia, en la tecnología y la comunicación, pero no se ha logrado erradicar, por desgracia, la violencia ni la guerra. Por esta razón, las Naciones Unidas han proclamado el año 2000 Año Internacional de la Cultura de Paz". ${ }^{36}$

El contenido del Manifiesto 2000 "Por una cultura de paz y no violencia" exige un compromiso que, en síntesis, se expresa de la siguiente manera:

- Respetar la vida y la dignidad de cada persona.

- Rechazar la violencia

- Compartir tiempo y recursos materiales. Cultivar la generosidad, evitar la exclusión, la injusticia y la opresión.

- Defender la libertad de expresión y la diversidad cultural.

- Promover un consumo responsable.

- Contribuir al desarrollo de la comunidad. ${ }^{37}$

Estamos sin duda ante valores sociales, notas positivas para la convivencia, para la participación democrática, válidas para construir un programa educativo de gran calidad. "Porque el Año 2000 debe ser un nuevo comienzo para todos nosotros. Juntos podemos transformar la cultura de guerra y de violencia en una cultura de paz y de no violencia"

"Porque esta evolución exige la participación de cada uno de nosotros y ofrece a los jóvenes y a las generaciones futuras valores que les ayuden a forjar un mundo más justo, más solidario, más libre, digno y armonioso, y con mejor prosperidad para todos"

"Porque la cultura de paz hace posible el desarrollo duradero, la protección del medio ambiente y la satisfacción personal de cada ser humano". No son necesarios comentarios específicos para descubrir que "cultura de paz y educación para la paz" se implican mutuamente.

¿Y si el año 2000 fuera un nuevo comienzo, la ocasión de transformar juntos la cultura de guerra y violencia por una cultura de paz y no violencia? Es la gran pre-

36. Mensaje del Director General de la UNESCO Kohichiro Matsuura, con motivo del Año Internacional de la Cultura de la Paz.

37. Página web de UNESCO.org. 
gunta con que se inicia la presentación de los seis puntos claves del Manifiesto. Puntos que están relacionados con valores, actitudes, comportamientos, nucleares todos ellos si queremos pensar en una educación para la paz.

Seis puntos que han sido firmados por Premios Nobel de la Paz y que cito a continuación: ${ }^{38}$

- Respetar todas las vidas.

- Rechazar la violencia.

- Liberar su generosidad.

- Escuchar para comprenderse.

- Preservar el Planeta.

- Reinventar la solidaridad.

Porque el año 2000 debe ser un nuevo comienzo para todos nosotros, juntos podemos transformar la cultura de guerra y de violencia en una cultura de paz y de no violencia.

\section{El año 2000, un año para la paz. Consideraciones finales}

La actualidad demuestra que el anhelado objetivo de paz y bienestar general aún están lejos: solidaridad y cooperación son más que nunca valores cardinales que la UNESCO promueve multiplicando y reforzando las relaciones educativas, científicas y culturales, con dos objetivos estrechamente vinculados. Por una parte el desarrollo que, superando el progreso material, debe responder a la realización de las aspiraciones humanas sin hipotecar el patrimonio de las generaciones futuras y por otra, el establecimiento de una cultura de paz, fundada en el aprendizaje de una ciudadanía consciente y plenamente ejercida.

El Año Internacional de la Cultura de Paz constituye una ocasión única para conceder todo su protagonismo al Proyecto "Hacia una cultura de la paz" y despertar tanto en los países como en las personas, el deseo entusiasta de participar activamente en la promoción de una paz duradera.

La intervención de la UNESCO es decisiva en estos ámbitos, alentando a los Estados Miembros y organizaciones externas a poner en marcha iniciativas de paz en los niveles local, nacional, regional e internacional en forma de programas educativos, que presten especial atención a los objetivos de los currículos, al contenido de los textos y otros materiales de educación, incluyendo las nuevas tecnologías junto con materiales de educación cívica, actividades culturales, iniciativas conjuntas con medios de comunicación social, y otras. ${ }^{39}$

38. Este mismo Manifiesto puede ser firmado por cada uno de nosotros con la fórmula: Me comprometo en mi vida cotodiana, en mi familia, mi trabajo, mi comunidad, mi país y mi región... (Ver página web de UNESCO.org/manifiesto2000).

39. Recordamos las numerosas Cátedras de la UNESCO por la paz, que pueden localizarse en Universidades de todo el mundo. 
Propone, en definitiva, que el año Internacional venga asociado a programas regionales e internacionales que insistan en el carácter prioritario de la paz, el desarrollo y la democracia incorporando al proceso las organizaciones intergubernamentales y las no gubernamentales. Además para promover la idea de una paz duradera, prevé dar continuidad a las actividades del Año Internacional más allá del 2000. Actualmente se están fortaleciendo estrechos vínculos de carácter internacional, nacional o local con interlocutores diversos.

En el Año 2001, Año Internacional de los Voluntarios y también Año del diálogo entre civilizaciones, se abrirán nuevas vías para crear relaciones de colaboración y proseguir diferentes actividades. En la propuesta que declaraba el Año 2000 "Año Internacional de la Cultura de la Paz" aparece como justificación decisiva, entre otras razones, la idea de que todo ello "estimularía los esfuerzos de la comunidad internacional para instaurar una cultura de paz dotada de carácter permanente". ${ }^{40}$

Este planteamiento supone, obviamente, "reemplazar la secular cultura de guerra por la de paz" conscientes de que se requiere un esfuerzo educativo continuado para modificar las maneras de reaccionar ante los problemas y para construir un desarroIlo sostenible que pueda suprimir las causas del conflicto. Grupos de expertos opinan que la clave es el desarrollo mutuo y sostenible sin el cual es imposible lograr una paz duradera, sin olvidar que el desarrollo mismo puede ser una fuente de conflicto en la medida en que se exige de la población el abandono de sus tradiciones y la reproducción de modelos de países desarrollados. Este hecho hace necesaria una revisión conceptual que incluya la dimensión humana y social, de modo que los pueblos puedan participar en los proyectos y así poner término al complejo de dependencia que engendra resentimiento y violencia. A la vez es preciso fomentar formas de comportamiento participativo, democrático, solidario y cooperativo que permita de manera adecuada y positiva gestionar los conflictos.

Termino con palabras del Mensaje antes citado, palabras autorizadas de Koichiro Matsuura: "La paz únicamente puede lograrse por nuestro comportamiento, nuestras actitudes y nuestro quehacer cotidiano. La cultura de paz es una cultura universal que todos los pueblos comparten y es consustancial a nuestra humanidad común. Construyamos juntos ese mundo de paz. Cultivemos juntos la paz... Que cada cual se pregunte qué puede hacer por la paz cada día". Es un gran desafío para la educación individual y social y es la mejor manera de construir el futuro, porque la paz no se garantiza sólo con acuerdos políticos, económicos o militares, depende más del compromiso unánime, sincero y constante de los pueblos. Cada uno de nosotros debe contribuir a crear un mundo en paz.

Los valores de la cultura de paz permiten diseñar un programa educativo excelente en el que los contenidos de los grandes apartados estén relacionados con eliminar la pobreza, reducir las desigualdades, lograr un desarrollo sostenible, respetar los derechos humanos, reforzar las instituciones democráticas, fomentar la libertad de expresión, mejorar la condición de la mujer, salvaguardar la diversidad cultural y el medio ambiente y, desde nuestra perspectiva, para lograr un mundo de paz y progre-

40. Proyecto Transdisciplinario, p. 4. 
so es preciso compartir el saber. El ejercicio responsable y oportuno del conocimiento ha de ir estrechamente vinculado a la necesidad de prevenir, a la capacidad de anticiparse a los hechos. Los enfoques parciales y de ámbito local deben superarse desde una visión global e interactiva que produzca mecanismos de anticipación y de prospectiva.

El reto de la educación y de la cultura de paz está por tanto en conceder responsabilidad a las personas para hacerlas protagonistas de su propia historia, y con instrumentos de transformación que no impliquen la destrucción u opresión ajena, y no transmitir intransigencia, odio y exclusión, puesto que ello siempre supondrá la anulación de nuestro propio proyecto de emancipación y desarrollo. ${ }^{41}$

Lo que está claro es que no nos basta con hacer un buen acopio de normas éticas y principios de conciencia, sino que es menester que todo esto se traduzca en cambios de conducta y en movilizaciones y creaciones culturales del 'vivir concreto y cotidiano, la cultura del pueblo' ${ }^{\prime 42}$ que permitan una transformación social, incluyendo nuestro propio comportamiento. Es preciso aprender a dar respuestas no violentas a los conflictos del tipo que sean, aplicar los principios desde los que construir una ética global o planetaria, tales como: el principio de reciprocidad, el principio de responsabilidad solidaria, el principio de equidad intergeneracional y el principio de sustentabilidad. ${ }^{43}$

Convencidos de la complejidad del tema parece evidente que "uno de los retos de la educación para la paz no puede ser otro que el participar en la formación de una ciudadanía dispuesta a abordar responsablemente los cambios estructurales que el mundo necesita, en lo político y en lo económico. Es un trabajo a largo plazo, de generaciones, pero que no permite dilación" ${ }^{44}$

41. V. Fisas, op. cit., p. 375.

42. M. Vidal, Postulados de una ética de la paz, Simposio Etica y cultura de paz, Madrid, diciembre 1985, p. 48.

43. V. Fisas, op. cit., pp. 363-364.

44. V. Fisas, op. cit., p. 366. 